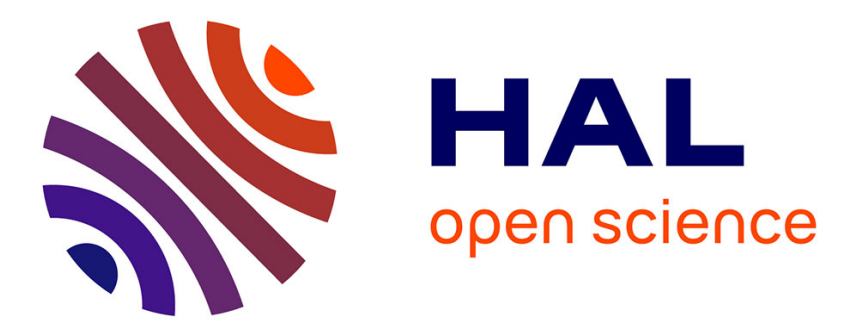

\title{
Ultranarrow and Wavelength-Tunable Thermal Emission in a Hybrid Metal-Optical Tamm State Structure
} Zhiyu Wang, J Kenji Clark, Ya-Lun Ho, Sebastian Volz, Hirofumi Daiguji, Jean-Jacques Delaunay

\section{- To cite this version:}

Zhiyu Wang, J Kenji Clark, Ya-Lun Ho, Sebastian Volz, Hirofumi Daiguji, et al.. Ultranarrow and Wavelength-Tunable Thermal Emission in a Hybrid Metal-Optical Tamm State Structure. ACS photonics, 2020, 7, pp.1569 - 1576. 10.1021/acsphotonics.0c00439 . hal-03011188

\section{HAL Id: hal-03011188 \\ https://hal.science/hal-03011188}

Submitted on 18 Nov 2020

HAL is a multi-disciplinary open access archive for the deposit and dissemination of scientific research documents, whether they are published or not. The documents may come from teaching and research institutions in France or abroad, or from public or private research centers.
L'archive ouverte pluridisciplinaire HAL, est destinée au dépôt et à la diffusion de documents scientifiques de niveau recherche, publiés ou non, émanant des établissements d'enseignement et de recherche français ou étrangers, des laboratoires publics ou privés. 
Subscriber access provided by Uppsala universitetsbibliotek

\section{Article}

\section{Ultra-narrow and wavelength-tunable thermal emission in a hybrid metal-optical Tamm state structure}

Zhiyu Wang, J. Kenji Clark, Ya-Lun Ho, Sebastian Volz, Hirofumi Daiguji, and Jean-Jacques Delaunay

ACS Photonics, Just Accepted Manuscript • DOI: 10.1021/acsphotonics.0c00439 • Publication Date (Web): 19 May 2020

Downloaded from pubs.acs.org on May 23, 2020

\section{Just Accepted}

"Just Accepted" manuscripts have been peer-reviewed and accepted for publication. They are posted online prior to technical editing, formatting for publication and author proofing. The American Chemical Society provides "Just Accepted" as a service to the research community to expedite the dissemination of scientific material as soon as possible after acceptance. "Just Accepted" manuscripts appear in full in PDF format accompanied by an HTML abstract. "Just Accepted" manuscripts have been fully peer reviewed, but should not be considered the official version of record. They are citable by the Digital Object Identifier (DOI®). "Just Accepted" is an optional service offered to authors. Therefore, the "Just Accepted" Web site may not include all articles that will be published in the journal. After a manuscript is technically edited and formatted, it will be removed from the "Just Accepted" Web site and published as an ASAP article. Note that technical editing may introduce minor changes to the manuscript text and/or graphics which could affect content, and all legal disclaimers and ethical guidelines that apply to the journal pertain. ACS cannot be held responsible for errors or consequences arising from the use of information contained in these "Just Accepted" manuscripts. 


\section{Ultra-narrow and wavelength-tunable thermal emission in a hybrid metal-optical Tamm state structure}

Zhiyu Wang, ${ }^{1}$ J. Kenji Clark, ${ }^{1}$ Ya-Lun Ho, ${ }^{1}$ Sebastian Volz, ${ }^{2,3}$ Hirofumi Daiguji, ${ }^{1}$ and Jean-Jacques Delaunay $^{1 *}$

${ }^{1}$ Department of Mechanical Engineering, School of Engineering, The University of Tokyo, Tokyo 113-8656, Japan

${ }_{2}^{2}$ Institute of Industrial Science and ${ }^{3}$ Laboratory for Integrated Micro Mechatronic

Systems/National Center for Scientific Research-Institute of Industrial Science (LIMMS/CNRSIIS), The University of Tokyo, Tokyo 153-8505, Japan

\section{Email list:}

wangzhiyu23@scale.t.u-tokyo.ac.jp

jkdclark@gmail.com

ho.ya-lun@scale.t.u-tokyo.ac.jp

volz@iis.u-tokyo.ac.jp

daiguji@thml.t.u-tokyo.ac.jp

*jean@mech.t.u-tokyo.ac.jp

TEL: +81-3-5841-3017 FAX: +81-3-5841-0441 


\begin{abstract}
Spectral selective thermal emitters are promising technological components due to their efficiency, large range of available emission wavelengths, simplicity, and long lifetime. Despite intensive effort into narrowband thermal emitters using surface plasmon polaritons, surface phonon polaritons, and Tamm plasmons, material losses have limited the potential quality factors, with the highest reported value being 200 . Here, by combining a metallic mirror and an optical Tamm state structure, we propose a hybrid structure that realizes narrowband and wavelength-tunable thermal emission. In the proposed structure, large energy can be stored between the loss-less DBRs $\left(\mathrm{SiO}_{2} / \mathrm{Ge}\right)$ thus drastically reducing metal losses. The metallic mirror is set at the bottom of the proposed structure to reduce the transmission losses and guarantee a large absorption/emission. This metal-optical Tamm state structure achieves experimentally a thermal emittance peak with a quality factor of over 750 at a wavelength of around $4.5 \mu \mathrm{m}$. Moreover, taking advantage of the temperature sensitivity of Ge, the thermal emission wavelength can be tuned by adjusting the operating temperature between 100 and $150{ }^{\circ} \mathrm{C}$. Both the high-quality factor and the wavelengthtunable properties demonstrate its suitability as a practical narrowband light source.
\end{abstract}

KEYWORDS: thermal emission, ultra-narrow bandwidth, wavelength-tunable, mid-infrared, optical Tamm state. 
The mid-infrared spectral range, where materials exhibit characteristic molecular absorption fingerprints originating from the intrinsic vibrational modes of chemical bonds, is crucial for biosensing ${ }^{(1),(2)}$ and gas detection. ${ }^{(3)}$ Conventional mid-infrared spectroscopy devices rely on broadband light sources with bulky gratings or interferometers in order to obtain spectrally resolved information. Miniaturized spectral selective emitters would allow for the design of spectroscopic devices without dispersive elements, drastically reducing their size and complexity.

Tailoring readily available thermal radiation into well-resolved and tunable emission at a single wavelength has been widely pursued as a means of creating low-cost and long-lifetime emitters with a large range of possible emission wavelengths. To date, wavelength-selective thermal emitters have been designed using a variety of technologies: photonic crystals utilizing band edge resonance, ${ }^{(4),(5)}$ surface plasmon/phonon polaritons (SPPs) excited by metallic/dielectric gratings, ${ }^{(6),(7)}$ localized surface plasmon resonances (LSPRs) realized by metamaterials, ${ }^{(8),(9)}$ and quantum-well inter-subband transition. ${ }^{(10),(11)}$ However, all of the above require two-dimensional (2D) or three-dimensional (3D) nanofabrication processes that are costly, and this hinders their practical use. Therefore, to realize narrowband emission over a large-area without increasing fabrication complexity, a planar multilayer structure based on surface-state resonances offers many advantages over 2D or 3D nanostructures.

In one-dimensional (1D) structures, a certain type of surface waves known as an optical Tamm state (OTS) can be sustained at the interface between two dielectric distributed Bragg reflectors (DBRs) with overlapping stopbands. ${ }^{(12)}$ As the in-plane wave vector of OTSs is smaller than the free space wave vector, OTSs can be excited from free space in a 1D structure without the need for additional nanostructures to couple light. As a consequence, lithography-free fabrication of OTS-based devices is possible. A class of OTS known as Tamm plasmons (TPs) 
was shown to exist at the interface between a DBR and a metallic film. In contrast to the high transmission of the OTS structures, ${ }^{(13)}$ the TP structures can efficiently absorb incident light within their metal film resulting in an absorption/emission peak. ${ }^{(14)}$ Using a TP structure configuration (i.e., a dielectric DBR on a metallic film) several narrowband thermal emitters with multilayer structures have been proposed. In 2017, a thermal emitter using a typical TP structure consisting of $\mathrm{Si} / \mathrm{SiO}_{2} \mathrm{DBR}$ and $\mathrm{Al}$ film underneath was shown to exhibit a quality factor $\left(Q\right.$-factor) over $30 .^{(15)}$ One year later, by inserting an optical cavity between a $\mathrm{Ge} / \mathrm{SiO}_{2} / \mathrm{Ge} \mathrm{DBR}$ and an $\mathrm{Au}$ film, we demonstrated a TP-cavity structure that had a thermal emittance peak with a $Q$-factor of 88 (16) $^{\text {In }}$ 2019 , deep learning method was used to optimize a $\mathrm{Ge} / \mathrm{SiO}_{2} \mathrm{DBR}$ on W film and was able to achieve a $Q$-factor as high as $188 .{ }^{(17)}$ Despite the efforts to improve the performance of spectrally selective thermal emitters based on TP structures, the $Q$-factors remain lower than the largest $Q$ factor of $\approx 200$ reported to date ${ }^{(7)}$ due to the large absorption losses induced by the metallic films. Without any metallic film, the OTS structure using two loss-less DBRs to store energy may offer a means to improve the $Q$ value of thermal emitters in a $1 \mathrm{D}$ configuration.

In addition to a narrowband emission peak, emission wavelength tunability is another requirement for a thermal emitter in real applications. Thermal emitters containing thermochromic materials such as $\mathrm{Ge}_{2} \mathrm{Sb}_{2} \mathrm{Te}_{5}(\mathrm{GST})$ and $\mathrm{VO}_{2}$ have been used to tune the emission wavelengths by controlling the operating temperatures. ${ }^{(18-20)}$ As these demonstrations rely on temperaturedependent phase transitions, they can only offer tunability over a specific temperature range. In addition, due to the relatively large loss introduced by these thermochromic materials, the thermal emitters usually cannot achieve high $Q$-factor (showing a $Q<10$ ) emission peaks. Achieving a narrowband and wavelength-tunable thermal emission with tunability over a wide range of wavelengths and operation at an arbitrary temperature remains challenging. 
In this work, we propose a $1 \mathrm{D}$ structure consisting of a $\mathrm{Ge} / \mathrm{SiO}_{2}$ OTS structure (i.e., $\mathrm{DBR}_{\text {top}}{ }^{-}$ $\mathrm{DBR}_{\text {bot }}$ ) and a Pt mirror (i.e., metal) as shown in Fig. 1a and realize narrow-band and wavelengthtunable thermal emission in the mid-infrared range. At the resonance wavelength, light is confined between the two loss-less DBRs of the hybrid metal-OTS structure leading to resonance with a high $Q$-factor (over 1000 is achieved through simulation) and the bottom metallic mirror blocks the light from leaking out thus providing an inelastic loss channel that guarantees a pronounced absorptance peak. Upon heating, a sharp peak is observed in the emittance spectrum with a $Q$ factor above 750, as shown in Fig. 1b. When this $Q$-factor is compared to that of other narrowband thermal emitters, it is superior to the LSPR structure by a factor of more than $55,{ }^{(8)}$ the QW ISBT structure by a factor of more than $10,{ }^{(11)}$ and the SPP structure and TP-based $1 \mathrm{D}$ structure by a factor of $\sim 4 .^{(7),(17)}$ Moreover, tuning of the emittance peak wavelength is also realized by taking advantage of the temperature sensitivity of $\mathrm{Ge}$, enabling fine adjustment of the emission wavelength. The emission peak wavelength can be tuned within a range of 4.6 times the bandwidth of the emittance peak by adjusting the temperature from 100 to $150{ }^{\circ} \mathrm{C}$. The tuning range to bandwidth ratio is larger than that of any other tunable thermal emitters which have been reported. ${ }^{(18-20)}$ Besides the outstanding wavelength selectivity and tunability, other properties such as polarization- and angle-independent emission for near-normal angles, and large device area make this hybrid metal-OTS structure a promising candidate for spectrally selective mid-infrared light sources.

\section{Results and discussion}

\section{Demonstrations and optimizations of the hybrid metal-OTS structure}


As illustrated in Fig. 1a, the two DBRs in the hybrid metal-OTS structure are adjacent to each other with Ge layer at the interface. The combination of $\mathrm{Ge}$ and $\mathrm{SiO}_{2}$ as the $\mathrm{DBR}$ layers provides a large refractive index contrast, resulting in the DBRs having a high reflectance in the midinfrared. The thickness of each DBR layer is set to have an optical thickness of one-quarter of the target wavelength $(4.5 \mu \mathrm{m})$. As a heat resistant material, Pt provides good thermal stability when used as the metallic layer at the bottom of the structure. The layer thicknesses of the different materials are fixed throughout this section, with $t_{\mathrm{Ge}}=280 \mathrm{~nm}, t_{\mathrm{SiO} 2}=819 \mathrm{~nm}$, and $t_{\mathrm{Pt}}=200 \mathrm{~nm}$. The hybrid metal-OTS structure exhibits a sharp and strong absorptance peak at normal incidence as shown in Fig. 1c (see Methods section for detailed information about simulation). The maximum of the absorptance peak is larger than 0.8 , and the peak has a $Q$-factor of 1908 , while at an off-resonance wavelength of $4.0 \mu \mathrm{m}$, the hybrid metal-OTS structure maintains a low absorptance of less than 0.001 , a value which is much lower than the absorptance of a semi-infinite Pt mirror (about 0.043 at the same wavelength used in the calculation). According to Kirchhoff's law, the emittance of a planar surface is equal to its absorptance, ${ }^{(21)}$ and therefore, this structure is expected to show not only a sharp and strong emission peak, but also low background emission over a large range of wavelengths.

The eigenmode of the hybrid metal-OTS structure can be studied using a similar method as described in (15). As shown in the inset in Fig. 1c, we separate the hybrid structure into a top reflector and a bottom reflector along a virtual interface (i.e., the interface of two DBRs illustrated by the white dashed line), and define the reflection coefficient of an upward-propagating wave at the virtual interface as $r_{\text {top }}$, and the reflection coefficient of a downward-propagating wave at the virtual interface as $r_{\text {bot, }}$ with the ambient being Ge in both cases. The confinement of light between the two reflectors requires 


$$
r_{\text {top }} r_{\text {bot }} \sim 1
$$

Here, $r_{\text {top }} r_{\text {bot }}$ as a function of wavelength is plotted in Fig. 1c (in pink color). The $r_{\text {top }} r_{\text {bot }}$ spectrum shows a peak with a near-unity value of 0.9992 corresponding to the same wavelength of the peak in the absorptance spectrum (in black color), suggesting that the strong and sharp absorptance peak is due to the high confinement of the eigenmode sustained at the interface between the top and the bottom reflectors. Without the bottom Pt mirror (i.e., for its corresponding OTS structure), the value of the $r_{\text {top }} r_{\text {bot }}$ peak would be only 0.9977 , showing that the addition of the Pt mirror enhances the confinement in the hybrid metal-OTS structure relative to a typical OTS structure.

To further compare the eigenmodes in the hybrid metal-OTS structure with the TP and OTS modes, we investigate the optical behaviors of TP, OTS, and hybrid metal-OTS structures for incident light from top (Figs. 1d and 1e). As shown by the normalized electric field $\left(|E| /\left|E_{0}\right|\right.$ ) distributions at the resonance wavelengths in Fig. 1d, the TP structure with the $\mathrm{Ge} / \mathrm{SiO}_{2} / \mathrm{Ge} \mathrm{DBR}$ and Pt mirror exhibits a relatively weak standing-wave pattern with a maximum normalized electric field value near the Pt mirror decaying towards the structure top, which corresponds to a relatively broad near-unity absorptance peak (the blue curve in Fig. 1e). Note that with only a three-layer DBR, this TP structure can sustain a fully developed TP mode. An increase in the number of the DBR pairs reduces its near-unity absorptance peak (see Fig. S1). Instead of using a metallic mirror to confine light, two DBRs having less material losses are employed in the OTS and hybrid metal-OTS structures. This results in improved light confinement observed as pronounced standing-wave patterns shown in the electric field distribution plots (Fig. 1d), thus revealing a much larger stored energy with sharper absorptance peaks (see the red and the black curves in Fig. 1e). Similar electric field distributions are found for the OTS and the metal-OTS 
structures, i.e., maximum values for the normalized electric field near the interface of the two DBRs (indicated as a white dashed line) decaying in intensity away from the interface. The nonzero electric field intensity below the bottom DBR of the OTS structure indicates that light transmits through the structure bottom, as evidenced by the transmittance peak at the resonance wavelength shown in the bottom-right plot of Fig. 1e. In contrast to the OTS structure, the metalOTS structure possesses low transmission losses. Indeed, the metal film at the structure bottom can not only improve the light confinement (evidenced by a stronger electric field distribution in Fig. 1d), but also reduce the transmission loss and provide an additional inelastic loss channel.(22) Accordingly, the hybrid metal-OTS structure exhibits an absorptance peak with a higher $Q$ value ( $Q$-factors are 1908 for the hybrid metal-OTS structure and 1324 for the OTS structure) and a twofold increase in the absorption intensity compared with that of the OTS structure (see the top-right plot in Fig. 1e). The zoomed-in plots red-rectangle-marked in Fig. 1d show absorption distributions around Pt mirror in TP and metal-OTS structures (here, absorption, noted Abs., is defined as $\omega \operatorname{Im}(\varepsilon$ )$|E(z)|^{2}$, where $\omega$ is the angular frequency, and $\operatorname{Im}(\varepsilon)$ is the imaginary part of permittivity) with logarithmic-scale. In the TP structure, most of the incident light is absorbed by the Pt mirror. This contrasts with the hybrid metal-OTS structure where most of the light is absorbed by the dielectric DBRs (see Fig. S2). The strong electric field of the DBRs results in two-order weaker light absorption in the Pt mirror. Interestingly, this weak metal absorption property of the metal-OTS structure is associated with a robust optical behavior (i.e., absorptance peak) independent of the metal used in the hybrid metal-OTS structure. Therefore, the metal is not limited to commonly used low-loss metals such as $\mathrm{Ag}, \mathrm{Au}$, and $\mathrm{Al}$, and a variety of metals could be chosen, such as $\mathrm{Ti}$ for good adhesion, Pt for good thermal stability, or Pd for catalysis, without any significant change in the optical behavior (see Fig. S3 and Table S1). Note that this is a consequence of the non-zero 
loss of the dielectric materials, as evidenced by the fact that when the dielectric loss is decreased to zero in simulation, the metal-dependent absorption will become obvious (see Fig. S3).

To optimize the optical behavior of the hybrid metal-OTS structure, we examine the absorptance spectra when the number of DBR layers is varied (Fig. 2a). Here, as demonstrated on the right part, the configuration of the $\mathrm{DBR}_{\mathrm{bot}}$ and the metallic film are fixed, and the layer number of $\mathrm{DBR}_{\text {top }}$ is varied ( $N_{\text {top }}=5,7$, and 9$)$. From $N_{\text {top }}=5$ to $N_{\text {top }}=7$, both the absorptance peak intensity and $Q$-factor improved. By adding two more layers (i.e., $N_{\text {top }}=9$ ), the peak intensity decreases drastically. As a result, the structure with the 7-layer DBR exhibits the best optical behavior when considering both absorptance intensity and $Q$ value. The optical behaviors change can be attributed to different optical "impedance matching" conditions for $N_{\text {top }}=5,7$ and 9. ${ }^{(15)} \mathrm{In}$ the case of $N_{\text {top }}=7$, it is found that the structure achieves the best impedance matching condition (the transmission loss through the top reflector matches the absorption loss of the lower reflector), thus generating a minimum reflection from the structure and a maximum for the absorptance peak.

The angular and polarization dependences are characterized for the hybrid metal-OTS structure with 7 layers in the $\mathrm{DBR}_{\text {top }}$. The simulated absorptance spectra for different incident angles of the transverse electric (TE-) and transverse magnetic (TM-) polarized light are shown in the left part of Fig. 2b. For both TE- and TM-polarized light, when the incident angle is small (e.g., within $5^{\circ}$ ), the wavelength deviation is relatively small (less than $3 \mathrm{~nm}$ ). However, for a larger incident angle, the resonance wavelength shows a larger angular dependence (e.g., the wavelength difference is as large as $47 \mathrm{~nm}$ between the case of normal incidence and the $20^{\circ}$ TM-polarized incidence). As the incident angle increases, TE-polarized spectra exhibit weaker absorptance peaks, while the TM-polarized spectra show stronger peaks. This intensity change can be explained by 
the difference in the impedance matching conditions occurring for different polarizations and incident angles. The right part of Fig. $2 b$ shows the wavelength difference $(|\Delta \lambda|)$ between the absorptance peaks of the TM and the TE polarizations for varying incident angles. The wavelength difference increases with the incident angle, but the difference caused by the different polarizations is relatively small compared with the peak resonance bandwidths (for the incident angle of $20^{\circ}$, the TM and TE show a wavelength difference of $1.2 \mathrm{~nm}$ while the bandwidth of the peak is around $2.5 \mathrm{~nm}$ ). In summary, the resonance wavelength of the hybrid metal-OTS structure exhibits a smaller angular and polarization dependence at small incident angles, so for the structure applied as thermal emitters, the emission near normal angle should be utilized to avoid a broadening of the emission spectrum.

\section{Tunability of the absorptance peak wavelength}

Figure 3a shows absorptance as a function of incident wavelength and the thickness of the middle Ge layer $t_{\text {mid }}$ (the absorptance is shown on a logarithmic-scale for a clear demonstration of the ultra-narrow absorptance peaks). High-absorptance lines represent resonance modes, and by varying $t_{\mathrm{mid}}$, the resonance wavelengths can be adjusted from $3.5 \mu \mathrm{m}$ to a wavelength longer than $5.5 \mu \mathrm{m}$. Therefore, the narrow absorptance peak can be artificially designed within a wide range of wavelengths by simply adjusting $t_{\text {mid. }}$. The inset of Fig. 3a shows the absorptance spectra for three different $t_{\text {mid }}$ values (i.e., $t_{\text {mid }}=0,560$, and $1120 \mathrm{~nm}$ ) as marked by the circles referring to different orders of the resonance modes. It is observed that higher-order modes have narrower bandwidths, allowing narrower bandwidth resonances to be designed without altering the DBR layer structure. 
In addition to the ability to design the absorptance peak wavelength by adjusting $t_{\text {mid }}$, the wavelength can also be actively tuned effect as a result of the temperature-dependent material properties. In Fig. 3b, we simulated the absorptance spectra of the hybrid structure with $\left(t_{\text {mid }}=\right.$ $1120 \mathrm{~nm})$ and without $\left(t_{\text {mid }}=0 \mathrm{~nm}\right)$ the middle Ge layer at different temperatures by considering the temperature dependence of the $\mathrm{SiO}_{2}$ and $\mathrm{Ge}$ refractive indices as well as the thermal expansion coefficients of $\mathrm{Ge}$ and $\mathrm{SiO}_{2}$. Using a linear fitting procedure, the thermo-optic coefficients of $\mathrm{Ge}$ and $\mathrm{SiO}_{2}$ are estimated to be $4.77 \times 10^{-4} /{ }^{\circ} \mathrm{C}$ and $1.23 \times 10^{-5 /}{ }^{\circ} \mathrm{C}$, respectively (see Fig. S4), ${ }^{(23),(24)}$ and the thermal expansion coefficients of $\mathrm{Ge}$ and $\mathrm{SiO}_{2}$ are taken directly from literatures as 5.90 $\times 10^{-6 /}{ }^{\circ} \mathrm{C}$ and $5.60 \times 10^{-7 /}{ }^{\circ} \mathrm{C}$, respectively. ${ }^{(25),(26)}$ Without the Ge middle layer, the simulated absorptance peak shows little variation with temperature, while for the structure with a thick Ge middle layer, a strong wavelength-tuning behavior is observed. As the temperature changes from 100 to $150^{\circ} \mathrm{C}$, the absorptance peak red-shifts by $23 \mathrm{~nm}$. The electric field distributions, shown to the right of the absorptance spectra, can be used to explain these different behaviors. For the structure without Ge middle layer, the maximum electric field exists in the $\mathrm{SiO}_{2}$ layer, while for the structure with a thick Ge middle layer, the maximum electric field is within the Ge layer. Because Ge has both a more than one-order larger thermo-optic coefficient and thermal expansion coefficient than $\mathrm{SiO}_{2}$, the structure with a Ge middle layer has a larger sensitivity to temperature. The middle Ge layer does not only store a large amount of energy, allowing for a high $Q$-factor resonance, but also acts as a wavelength tuning material. This structure with a thick middle Ge layer $\left(t_{\mathrm{mid}}\right.$ around $\left.1120 \mathrm{~nm}\right)$ was therefore chosen for fabrication.

\section{Experimental results}


The hybrid metal-OTS structure was fabricated and its reflectance and emittance spectra were measured as shown in Fig. 4 (see Methods section for the detailed information about the fabrication and the measurement). As shown in Fig. 4a, the measured reflectance spectrum under normal incidence $\left(0^{\circ}\right)$ shows a sharp dip at $4.516 \mu \mathrm{m}$ with a bandwidth of $4.7 \mathrm{~nm}$ (corresponding to a $Q$ factor of 961). The reflectance dip blue-shifts by only $1.4 \mathrm{~nm}$ when the incident angle is varied from $0^{\circ}$ to $5^{\circ}$ under transverse-electric (TE) polarized incidence. An additional $5.4 \mathrm{~nm}$ shift is observed when the incident angle is further increased from $5^{\circ}$ to $10^{\circ}$. This angular dependence reveals a relatively robust optical behavior for near-normal incidence (e.g., within $\pm 5^{\circ}$ ). More information about the angular and polarization dependence is provided in the Supporting Information (Fig. S5). In Fig. 4(b), the simulations are performed for different incident angles (TEpolarized) with the actual layer thicknesses determined by cross-section scanning electron microscopy (inset in Fig. 4a). The wavelength of the reflectance dips matches well with the measured spectra of the fabricated structure. The deviations in the modulation depth and bandwidth may be due to imperfections in the deposited dielectric layers introducing a larger optical loss (see Fig. S6).

Figure $4 \mathrm{c}$ shows the measured emission spectra of the structure for varying temperatures between 100 and $150^{\circ} \mathrm{C}$. As temperature rises, in addition to the intensity increasing, the emission peak shifts to longer wavelengths. After normalizing by the emission of a blackbody, the emittance spectra of the structure for different temperatures are plotted in Fig. 4d. The room temperature emittance (grey colored, calculated as 1 - reflectance) is shown as a reference. The red-shift of the emittance peak wavelength with increasing temperature is observed. For every $10{ }^{\circ} \mathrm{C}$ increase in temperature, the wavelength of the emittance peak red-shifts by about $5.4 \mathrm{~nm}$, with a total change from 100 to $150{ }^{\circ} \mathrm{C}$ of $27.1 \mathrm{~nm}$. The shift of the emittance peak is 4.6 times the average bandwidth 
$(5.9 \mathrm{~nm})$ of the measured emittance peaks. Based on the data of thermo-optic coefficients of Ge and $\mathrm{SiO}_{2}$ and their thermal expansion coefficients, ${ }^{(23-26)}$ the trend of the emittance wavelength shift can be reproduced by simulation (see Fig. S7). Note that this wavelength tuning behavior is not caused by the thermal degradation of the sample, as can be confirmed by the reflectance spectra taken before and after thermal emission measurements (see Fig. S8). The emittance spectra for different temperatures show similar maximum emittance values about 0.35 , and the $Q$-factors of these emittance peaks are 789,974, 762, 775, 703, and 684 (average $Q$-factor of 780) for temperatures from 100 to $150^{\circ} \mathrm{C}$. Compared with the room temperature emittance, the emittance peaks at elevated temperatures exhibit smaller $Q$-factors and modulations, which should be attributed to increased losses in each layer material due to the elevated temperatures. Nonetheless, the $Q$-factor and tunable range vs. bandwidth ratio are superior to those of any other reported thermal emitters (see the list in Table 1). This temperature sensitive narrowband thermal emitter is expected to be used as a finely tunable monochromatic light source for the analysis of characteristic molecular absorptions in applications such as volatile vapor identification and biosensing.

\section{Conclusions}

A hybrid metal-OTS structure with optimized layer thicknesses was investigated as a wavelengthselective and wavelength-tunable thermal emitter in the mid-infrared range. The OTS features allow the structure to store a large amount of energy, resulting in a high- $Q$-factor resonance, while the metallic mirror helps to avoid light leakage, guaranteeing a large absorption at the resonance wavelength. The structure is optimized for a narrow bandwidth and highly tunable absorptance peak under normal incidence. The hybrid structure was fabricated, and ultra-narrow emission 
peaks with an average $Q$-factor of 780 were observed, representing a 4 -fold increase over the best experimental results reported so far. Additionally, by taking advantage of the large thermo-optic and thermal expansion coefficients of Ge, the sharp emittance peak was tuned over a range as large as 4.6 times the emittance peak bandwidth. This work demonstrates the possibility of achieving narrowband and tunable thermal emitters in the mid-infrared for use as light sources in chemicaland bio-sensing.

\section{Materials and methods}

\section{Simulation details}

The simulated reflectance, absorptance, and transmittance spectra, and the near-field electric field magnitude distributions were computed using rigorous coupled-wave analysis (DiffractMOD, Rsoft Design Group, Ossining, USA). The results for $r_{\text {top }} r_{\text {bot }}$ as a function of wavelength were calculated using the transfer-matrix method. The complex permittivities of Pt and Ti are described by the Drude-Lorentz dispersion model. ${ }^{(29)}$ The refractive indices of $\mathrm{Ge}^{(30)}$ and $\mathrm{SiO}_{2}{ }^{(31)}$ are modeled according to literature values.

\section{Fabrication details}

The thin films were deposited on a fused quartz substrate using an RF-magnetron sputtering system (CFS-4ES, Shibaura Engineering Works Co., Ltd., Yokohama, Japan). First, a $200 \mathrm{~nm}$ Pt layer is deposited with a $10 \mathrm{~nm}$ Ti layer underneath as an adhesion layer. Then, a $\mathrm{SiO}_{2}$ layer is deposited 
with a $5 \mathrm{~nm} \mathrm{Ti}$ adhesion layer. Afterward, the rest $\mathrm{Ge} / \mathrm{SiO}_{2}$ layers were deposited. Finally, an additional $\mathrm{SiO}_{2}$ layer was deposited on the top of the structure as a protection layer (see Fig. S9 for detailed information about optical behavior with and without the protection layer). All the RF sputtering processes were done with Ar at a flowing rate of $13 \mathrm{sccm}$ and a pressure of 0.6-0.7 $\mathrm{Pa}$. After the deposition, the sample was annealed in air at $150{ }^{\circ} \mathrm{C}$ for 6 hours to reduce the internal stress.

\section{Measurement details}

The reflectance spectra of the fabricated structures were measured with incident angle of $0^{\circ}, 5^{\circ}$, and $10^{\circ}$ using an FT-IR spectrometer (VIR-300, JASCO Co., Tokyo, Japan) with a spectral data spacing of $0.241 \mathrm{~cm}^{-1}$, and calibration was done with an Au mirror (see Fig. S10). To measure the thermal emission of the fabricated hybrid structure, the sample was heated up to $150{ }^{\circ} \mathrm{C}$ using a heating stage (UH200CV, SPLEAD Co., Tokyo, Japan). Emission from the sample was measured through the external port of a Thermo Nicolet iS50 FT-IR (THERMO FISHER SCIENTIFIC Co., Waltham, MA, USA) with a spectral data spacing of $0.241 \mathrm{~cm}^{-1}$. A carbon soot film was deposited on a Si substrate to act as a blackbody reference. The surface temperature of the fabricated structure was measured using a K-type thermocouple equipped with a surface temperature probe (IK-500, AS ONE Corp., Osaka, Japan); the temperature of the carbon soot surface was determined using an infrared thermometer (IT-545S, HORIBA Ltd., Kyoto, Japan). 
Supporting Information: Absorptance spectra of the TP structure with additional DBR pairs, the electric field and absorption distributions of the hybrid metal-OTS structure at the absorptance peak wavelength for normal incidence, the hybrid metal-OTS structure with different metal layers, the fitting procedure of the thermo-optic coefficients for $\mathrm{Ge}$ and $\mathrm{SiO}_{2}$, the simulated absorptance dispersion diagrams of the fabricated structure, the simulated spectrum with increased dielectric material losses, the simulated trend of the emittance wavelength shift, the stability test showing the reflectance spectrum after the emission measurements, the reflectance spectra with and without the top $\mathrm{SiO}_{2}$ protection layer, and the calibration of the measured reflectance spectrum.

\section{Acknowledgments}

This work was supported through Japan Society for the Promotion of Science (JSPS) KAKENHI Grant Numbers 17H03229 and 19J13668, JSPS CREST (JPMJCR17I3), and Leadership Development Program for Ph.D. (LDPP), The University of Tokyo. This work was conducted at Takeda Sentanchi Supercleanroom, The University of Tokyo, supported by "Nanotechnology Platform Program" of the Ministry of Education, Culture, Sports, Science and Technology (MEXT), Japan, Grant Number JPMXP09F-19-UT-0129. We would like to extend our grateful appreciation to Ms. Ayako Mizushima and Dr. Eric Lebrasseur from The University of Tokyo for important technical support.

\section{Conflict of interest}


The authors declare that they have no conflict of interest.

\section{Contributions}

J.-J.D. supervised the entire project with Y.-L.H. Z.W. fabricated the samples, performed the experiments and analysed the data. J.-J.D., Z.W., J.K.C., Y.-L.H., S.V. and H.D. discussed the results and wrote the paper.

\section{References}

(1) Lin-Vien, D.; Colthurp, N. B.; Fateley, W. G.; Grasselli, J. G. The Handbook of Infrared and Raman Characteristic Frequencies of Organic Molecules; Academic Press, 1991.

(2) Kaplan, H. Practical Applications of Infrared Thermal Sensing and Imaging Equipment; SPIE Press, 2007.

(3) Chen, W.; Mouret, G.; Boucher, D.; Tittel, F. K. Mid-infrared trace gas detection using continuous-wave difference frequency generation in periodically poled $\mathrm{RbTiOAsO}_{4}$. Appl. Phys. B: Lasers Opt. 2001, 72, 873-876.

(4) Celanovic, I.; Jovanovic, N.; Kassakian, J. Two-dimensional tungsten photonic crystals as selective thermal emitters. Appl. Phys. Lett. 2008, 92, 193101. 
(5) Rinnerbauer, V.; Yeng, Y. X.; Chan, W. R.; Senkevich, J. J.; Joannopoulos, T. D.; Soljačić, M.; Celanovic, I. High-temperature stability and selective thermal emission of polycrystalline tantalum photonic crystals. Opt. Express 2013, 21, 11482-11491.

(6) Liu, J.; Guler, U.; Lagutchev, A.; Kildishev, A.; Malis, O.; Boltasseva, A.; Shalaev, V.M. Quasi-coherent thermal emitter based on refractory plasmonic materials. Opt. Mater. Express 2015, 5, 2721-2728.

(7) Dahan, N.; Niv, A.; Biener, G.; Gorodetski, Y.; Kleiner, V.; Hasman, E. Extraordinary coherent thermal emission from $\mathrm{SiC}$ due to coupled resonant cavities. J. Heat Transfer 2008, 130, 112401.

(8) Dao, T. D.; Chen K.; Ishii, S.; Ohi, A.; Nabatame, T.; Kitajima, M.; Nagao, T. Infrared perfect absorbers fabricated by colloidal mask etching of $\mathrm{Al}-\mathrm{Al}_{2} \mathrm{O}_{3}-\mathrm{Al}$ trilayers. $A C S$ Photonics 2015, 2, 964-970.

(9) Liu, X.; Tyler, T.; Starr, T.; Starr, A. F.; Jokerst, N. M.; Padilla, W. J. Taming the blackbody with infrared metamaterials as selective thermal emitters. Phys. Rev. Lett. 2011, 107, 045901.

(10) De Zoysa, M.; Asano, T.; Mochizuki, K.; Oskooi, A.; Inoue, T.; Noda, S. Conversion of broadband to narrowband thermal emission through energy recycling. Nat. Photonics 2012, 6, 535 .

(11) Inoue, T.; De Zoysa, M.; Asano, T.; Noda, S. Realization of dynamic thermal emission control. Nat. Mater. 2014, 13, 928. 
(12) Kavokin, A. V.; Shelykh, I. A.; Malpuech, G. Lossless interface modes at the boundary between two periodic dielectric structures. Phys. Rev. B 2005, 72, 233102.

(13) Goto, T.; Dorofeenko, A. V.; Merzlikin, A. M.; Baryshev, A.V.; Vinogradov, A. P.; Inoue, M.; Lisyansky, A. A.; Granovsky, A. B. Optical Tamm states in one-dimensional magnetophotonic structures. Phys. Rev. Lett. 2008, 101, 113902.

(14) Kaliteevski, M.; Iorsh, I.; Brand, S.; Abram, R. A.; Chamberlain, J. M.; Kavokin, A. V.; Shelykh, I. A. Tamm plasmon-polaritons: Possible electromagnetic states at the interface of a metal and a dielectric Bragg mirror. Phys. Rev. B 2007, 76, 165415.

(15) Yang, Z.-Y.; Ishii, S.; Yokoyama, T.; Dao, T. D.; Sun, M.-G.; Pankin, P. S.; Timofeev, I. V.; Nagao T.; Chen, K.-P. Narrowband wavelength selective thermal emitters by confined Tamm plasmon polaritons. ACS Photonics 2017, 4, 2212-2219.

(16) Wang, Z.; Clark, J. K.; Ho, Y.-L.; Vilquin, B.; Daiguji, H.; Delaunay, J.-J. Narrowband thermal emission realized through the coupling of cavity and Tamm plasmon resonances. ACS Photonics 2018, 5, 2446-2452.

(17) Sakurai, A.; Yada, K.; Simomura, T.; Ju, S.; Kashiwagi, M.; Okada, H.; Nagao, T.; Tsuda, K.; Shiomi, J. Ultranarrow-band wavelength-selective thermal emission with aperiodic multilayered metamaterials designed by Bayesian optimization. ACS Cent. Sci. 2019, 5, 319-326.

(18) Du, K.-K.; Li, Q.; Lyu, Y.-B.; Ding, J.-C.; Lu, Y.; Cheng, Z.-Y.; Qiu, M. Control over emissivity of zero-static-power thermal emitters based on phase-changing material GST. Light: Sci. Appl. 2017, 6, e16194. 
(19) Kats, M. A.; Blanchard, R.; Zhang, S.; Genevet, P.; Ko, C.; Ramanathan, S.; Capasso, F. Vanadium dioxide as a natural disordered metamaterial: perfect thermal emission and large broadband negative differential thermal emittance. Phys. Rev. X 2013, 3, 041004.

(20) Du, K.; Cai, L.; Luo, H.; Lu, Y.; Tian, J.; Qu, Y.; Ghosh, P.; Lyu, Y.; Cheng, Z.; Qiu, M.; Li, Q. Wavelength-tunable mid-infrared thermal emitters with a non-volatile phase changing material. Nanoscale 2018, 10, 4415-4420.

(21) Siegel, R.; Howell, J. Thermal Radiation Heat Transfer; Taylor \& Francis: New York, 2002.

(22) Li, Z.; Butun, S.; Aydin, K. Large-area, lithography-free super absorbers and color filters at visible frequencies using ultrathin metallic films. ACS Photonics 2015, 2, 183-188.

(23) Li, H. H. Refractive index of silicon and germanium and its wavelength and temperature derivatives. J. Phys. Chem. Ref. Data 1980, 9, 561.

(24) Tan, C. Z.; Arndt, J. J. Temperature dependence of refractive index of glassy SiO2 in the infrared wavelength range. Phys. Chem. Solids 2000, 61, 1315.

(25) Kagaya, H.-M.; Soma, T. Temperature dependence of the linear thermal expansion coefficient for Si and Ge. Phys. Stat. Sol. (b) 1985, 129, K5.

(26) El-Kareh, B. Fundamentals of Semiconductor Processing Technologies (Kluwer Academic Publisher, Norwell, 1995).

(27) Cao, T.; Zhang, X.; Dong, W.; Lu, L.; Zhou, X.; Zhuang, X.; Deng, J. Tuneable thermal emission using chalcogenide metasurface. Adv. Opt. Mater. 2018, 6, 1800169. 
(28) Zhu, H.; Luo, H.; Li, Q.; Zhao, D.; Cai, L.; Du, K.; Xu, Z. Tunable narrowband midinfrared thermal emitter with a bilayer cavity enhanced Tamm plasmon. Opt. Lett. 2018, $43,5230-5233$.

(29) Rakić, A. D.; Djurišić, A. B.; Elazar, J. M.; Majewski, M. L. Optical properties of metallic films for vertical-cavity optoelectronic devices. Appl. Opt. 1998, 37, 5271-5283.

(30) Palik, E. D. Ed. In Handbook of Optical Constants of Solids; Academic Press, 1998; Vol. 3.

(31) Kischkat, J.; Peters, S.; Gruska, B.; Semtsiv, M.; Chashnikova, M.; Klinkmüller, M.; Fedosenko, O.; Machulik, S.; Aleksandrova, A.; Monastyrskyi, G.; Flores, Y.; Masselink, W. T. Mid-infrared optical properties of thin films of aluminum oxide, titanium dioxide, silicon dioxide, aluminum nitride, and silicon nitride. Appl. Opt. 2012, 51, 6789-6798. 

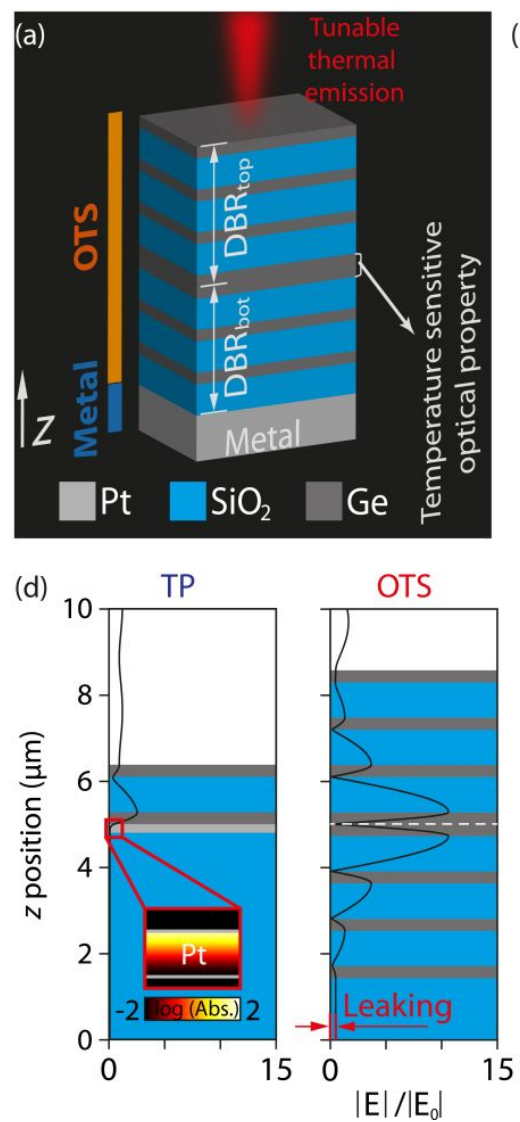
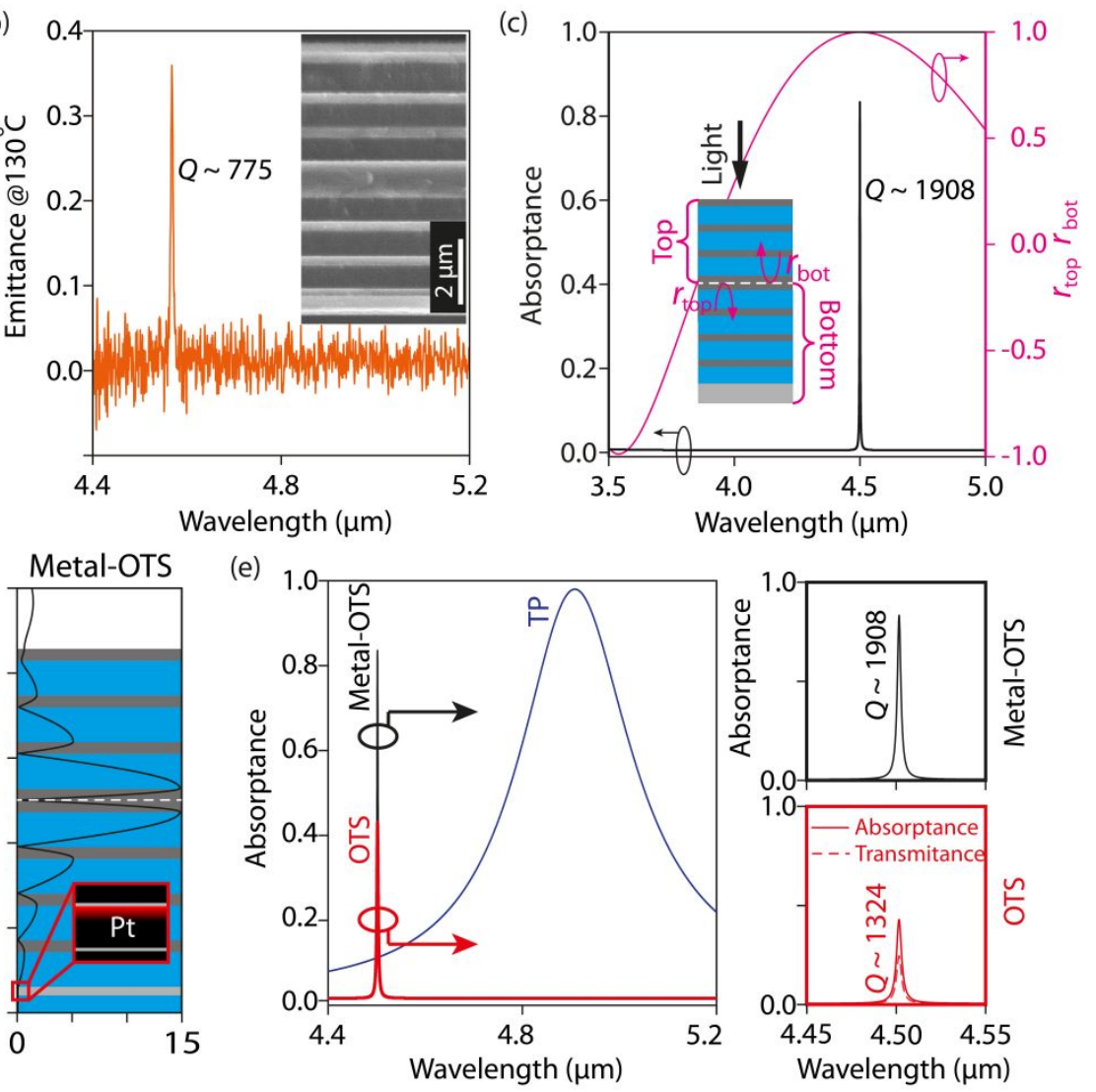

Figure 1. (a) Illustration of the hybrid metal-OTS structure as a wavelength-tunable thermal emitter. (b) Measured emittance spectrum at $130{ }^{\circ} \mathrm{C}$. (c) Simulated absorptance spectrum (in black color) for the hybrid metal-OTS structure under normal incident light with $t_{\mathrm{Ge}}=280 \mathrm{~nm}, t_{\mathrm{SiO} 2}=$ $819 \mathrm{~nm}$, and $t_{\mathrm{Pt}}=200 \mathrm{~nm}$ (here, $t_{\mathrm{Ge}}, t_{\mathrm{SiO} 2}$, and $t_{\mathrm{Pt}}$ are the thicknesses of the $\mathrm{Ge}, \mathrm{SiO}_{2}$, and Pt layers, respectively); the calculated $r_{\text {top }} r_{\text {bot }}$ as a function of wavelength (in pink color) ( $r_{\text {top }}$ and $r_{\text {bot }}$ are the reflection coefficients of a wave incident on the virtual interface, indicated as the white dashed line, with different directions as shown in the inset). (d) The simulated normalized electric field magnitude $\left(|E| /\left|E_{0}\right|\right)$ distributions of the TP, the OTS, and the hybrid metal-OTS structures at the wavelengths of the absorptance peaks ( $\left|E_{0}\right|$ is the incident electric field magnitude). The zoomedin plots with their absorption distributions along the Pt mirror are shown. (e) The absorptance spectra of the TP, OTS, and hybrid metal-OTS structures for normal incidence. The zoom-in plots for the absorptance spectrum of the hybrid metal-OTS structure, and the absorptance and transmittance spectra of the OTS structure are shown on the right side. 
2

3

4

5

6

7

8

9

10

11

12

13

14

15

16

17

18

19

20
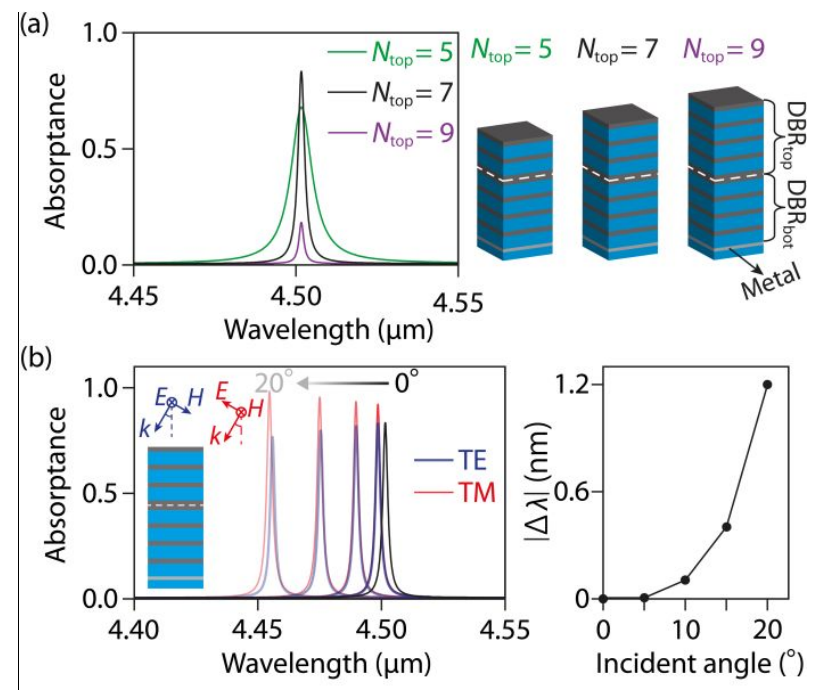

Figure 2. (a) Simulated absorptance spectra for the hybrid metal-OTS structures with different layer numbers $\left(N_{\text {top }}\right)$ in the $\mathrm{DBR}_{\text {top }}$ under normal incidence, with schematic diagrams shown on the right side. (b) Left: Simulated absorptance spectra for the hybrid metal-OTS structures with $N_{\text {top }}=$ 7 for different polarizations and incident angles of light; right: the wavelength differences between the TE- and TM-polarizations calculated for different incident angles. 

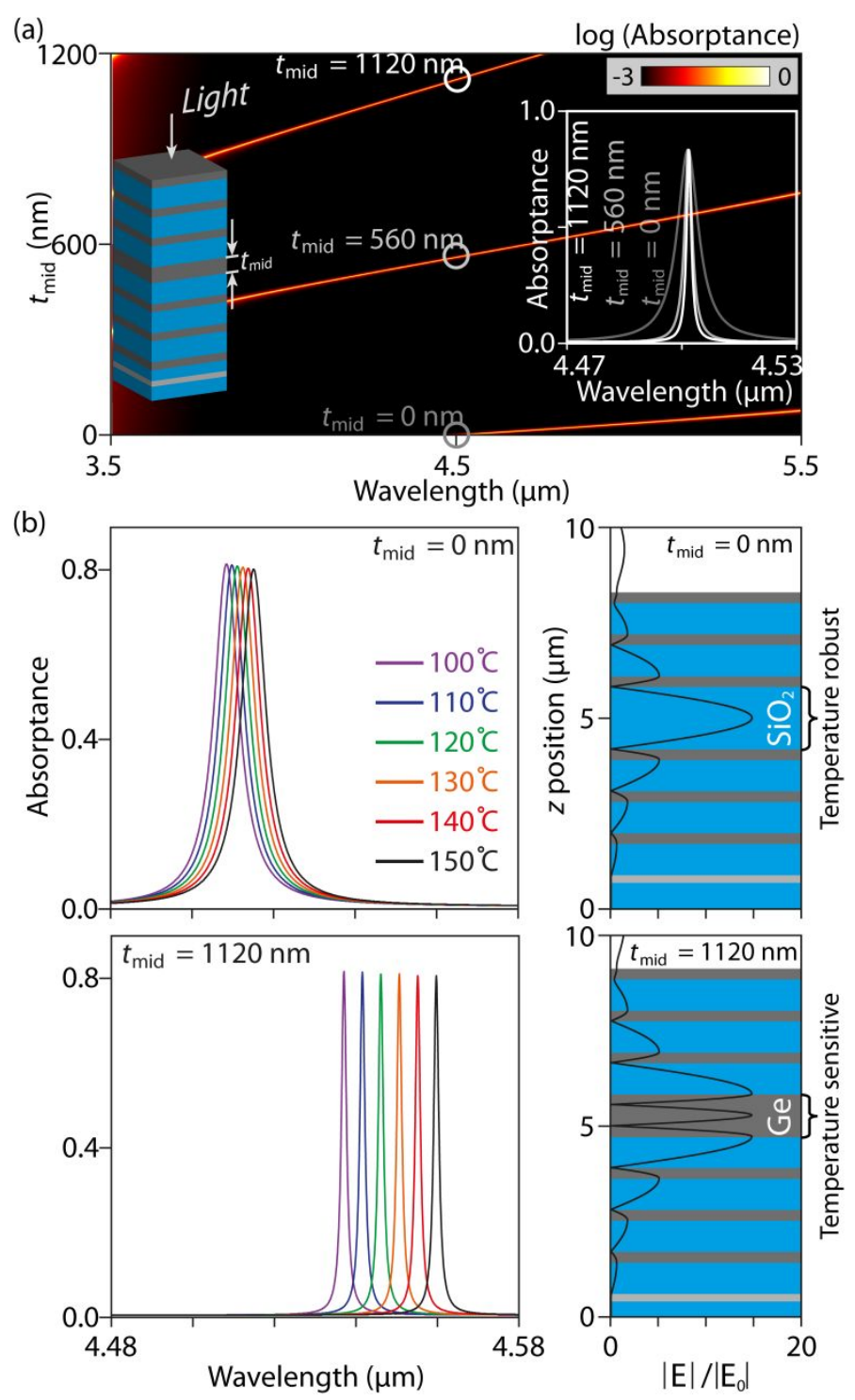

Figure 3. (a) The simulated absorptance varying with light wavelength and thickness of the middle Ge layer $t_{\text {mid }}$ under normal incident light on a logarithmic-scale. The absorptance spectra for the three $t_{\text {mid }}$ thicknesses are exhibited in the inset on the right side (the structure for $t_{\text {mid }}=560 \mathrm{~nm}$ is identical to the hybrid metal-OTS structure discussed in Fig. 1 and Structure 4 in Fig. 2. (b) The simulated absorptance spectra of the structures with $t_{\text {mid }}=0$ and $1120 \mathrm{~nm}$ at temperatures from 100 to $150{ }^{\circ} \mathrm{C}$, together with their normalized electric field magnitude distributions at the wavelengths of their absorptance peaks at room temperature. 
2

3

4

5

6

7

8

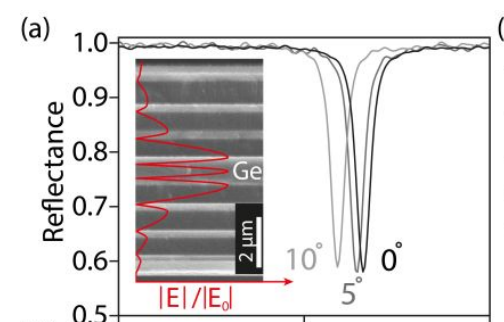

(b)
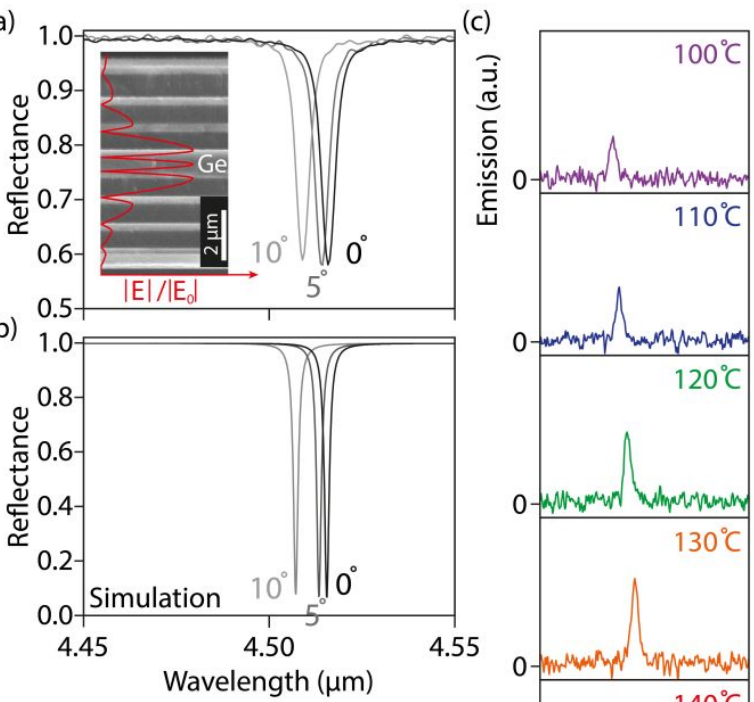

(d)

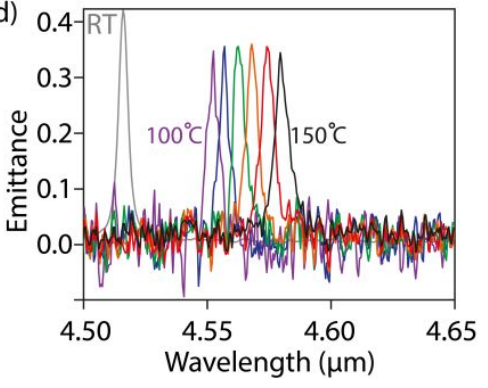

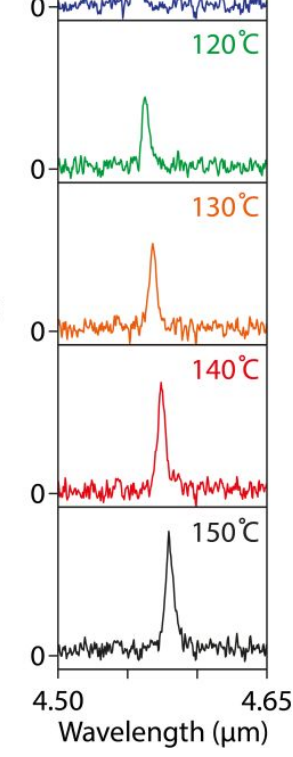

Figure 4. Measured (a) and simulated (b) reflectance spectra of the fabricated hybrid metal-OTS structure for three different incident angles $\left(0,5\right.$, and $\left.10^{\circ}\right)$ under TE-polarized incident light. The actual thicknesses of the fabricated device were determined by scanning electron microscopy cross-sectional view in the inset, where the red curve reveals the normalized electric field distribution according to the actual dimension. (c) Emission spectrum of the fabricated hybrid metal-OTS structure at different temperatures of 100, 110, 120,130, 140, and $150{ }^{\circ} \mathrm{C}$. (d) Emittance spectra of the fabricated hybrid metal-OTS structure at different temperatures including the room temperature (marked as "RT" in grey color) shown as a reference. 


\section{Tables}

Table 1. A summary of recently reported thermal emitters 
For Table of Contents Use Only

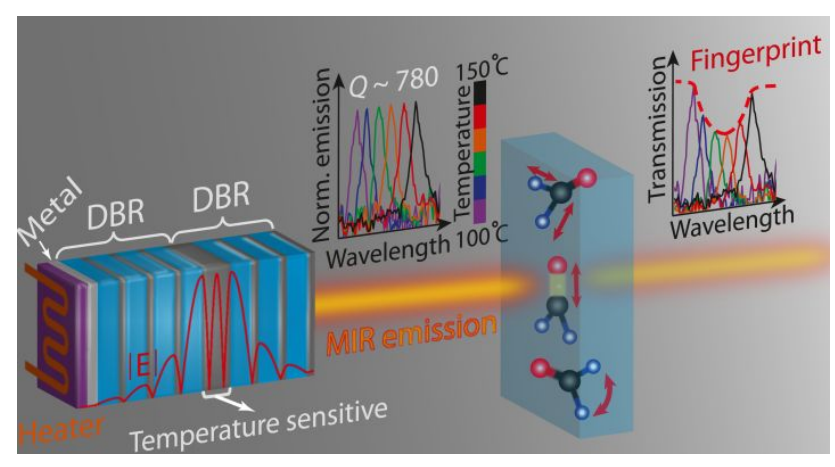

TOC Graphic. 3D schematic diagram of a portable spectroscopy device realized with the proposed thermal emitter that enables narrowband and wavelength-tunable emission in the midinfrared (MIR) range. The thermal emitter consisting of different functional components indicated in white (i.e., DBR and Pt layer) is shown with the superimposed electric field distribution (red curve) at the resonance wavelength. Taking advantage of the large field confinement and temperature-sensitive middle Ge layer, a narrowband and wavelength-tunable thermal emission is realized by controlling the operating temperature. The "normalized emission" spectra are based on the measurement results. By measuring the transmission through the formaldehyde sample at different operating temperatures, the fingerprint of formaldehyde (e.g., $\mathrm{C}=\mathrm{O}$ stretch as shown in the TOC) can be detected. Since the emission peak is more than one order sharper than the fingerprint of $\mathrm{C}=\mathrm{O}$ stretch in formaldehyde (the quality factor of emission peak is about 780, while the quality factor of the fingerprint is less than 70), the fingerprint can be detected without the need of optical filters or gratings, thus realizing a portable spectroscopy device. Moreover, the proposed thermal emitter is fabricated by conventional layer deposition methods (e.g., sputtering), enabling a large area and low-cost monochromatic light source in the MIR range. 